\title{
Genomics of Squamous Cell Lung Cancer
}

\author{
Melissa Rooney, ${ }^{\mathrm{a}, \mathrm{b}}$ SidDhartha DeVARAKonda, ${ }^{\mathrm{c}}$ RAmASWAMy Govindan ${ }^{\mathrm{a}, \mathrm{b}}$ \\ ${ }^{a}$ Division of Oncology, Department of Medicine and ${ }^{b}$ Alvin J. Siteman Cancer Center, Washington University School of Medicine, St. Louis, \\ Missouri, USA; 'Department of Internal Medicine, St. Luke's Hospital, Chesterfield, Missouri, USA \\ Disclosures of potential conflicts of interest may be found at the end of this article.
}

Learning Objectives

Describe important molecular aberrations associated with squamous cell carcinoma.

Identify molecular aberrations that may have therapeutic implications.

\begin{abstract}
Approximately $30 \%$ of patients with non-small cell lung cancer have the squamous cell carcinoma (SQCC) histological subtype. Although targeted therapies have improved outcomes in patients with adenocarcinoma, no agents are currently approved specifically for use in SQCC. The Cancer Genome Atlas (TCGA) recently published the results of comprehensive genomic analyses of tumor samples from 178 patients with

SQCC of the lung. In this review, we briefly discuss key molecular aberrations reported by TCGA and other investigators and their potential therapeutic implications. Carefully designed preclinical and clinical studies based on these largescale genomic analyses are critical to improve the outcomes of patients with SQCC of lung in the near future. The Oncologist 2013;18:707-716
\end{abstract}

Implications for Practice: Research conducted over the past few decades has provided crucial insights into the molecular pathogenesis of squamous cell carcinoma (SQCC) of the lung. The ongoing efforts by The Cancer Genome Atlas (TCGA) to comprehensively characterize the genomic landscape of SQCC of the lung has provided new impetus to develop innovative therapeutic approaches.

\section{INTRODUCTION}

Lung cancer is the leading cause of cancer-related mortality in the United States and worldwide [1, 2]. It is estimated that lung cancer will account for more than $25 \%$ of cancer-related deaths in the United States in the year 2013 [3]. Non-small cell lung cancer (NSCLC) comprises approximately $85 \%$ of all lung cancer diagnoses $[4,5]$. Squamous cell carcinoma (SQCC) represents approximately $20 \%-30 \%$ of NSCLC cases [6]. Histologically, SQCC is characterized by the presence of keratinization in the form of squamous pearls and intercellular bridges. Similar to cervical cancer, it is thought that SQCCs of the lung develop through a process of dysplasia, progressing into invasive cancers over several years [7]. The World Health Organization currently recognizes four variants of SQCC based on the histological appearance (papillary, clear cell, small cell, and basaloid), although the clinical significance of this classification remains uncertain [6].

The prognosis of patients with metastatic SQCC of the lung continues to be dismal. It is clear that cytotoxic chemotherapy regimens produce only limited benefit in this setting. Novel approaches are urgently needed to significantly improve the outcomes. The massive effort by The Cancer Genome Atlas (TCGA) to understand the genomic landscape of malignant tumors, including SQCC of the lung, has provided insight into several crucial signaling pathways that contribute to the pathogenesis of cancer. Sixty-four percent of the samples analyzed by TCGA were considered to harbor potentially targetable genetic alterations (Fig. 1) [8]. A number of preclinical and clinical studies are required to translate these exciting findings to improve the overall survival of patients with SQCC of the lung. We review here the findings from the TCGA and other groups that provide rationale for developing innovative clinical trials.

\section{MOLECUlar Alterations in SQuAMOUS Cell LUNG CANCER}

\section{Chromosomal Level Alterations}

Allelic losses that involve loci containing tumor suppressor genes or gains involving selective chromosomal regions that include oncogenes predispose cells to malignant transformation [9]. Haploinsufficiency or partial inactivation of tumor suppressor genes also contributes to tumorigenesis $[9,10]$. The most frequently reported sites of allelic loss in SQCC of the lung involve chromosomes 3, 5, 9, 13, and 17 [11-13]. Many of these regions carry certain known tumor suppressor genes, such as TP53 (17p), RB1 (13q), and APC (5q). In the context of SQCC, amplification of $3 q$ and allelic losses on chromosomal regions $3 p$ and $9 p$ are of particular interest. 


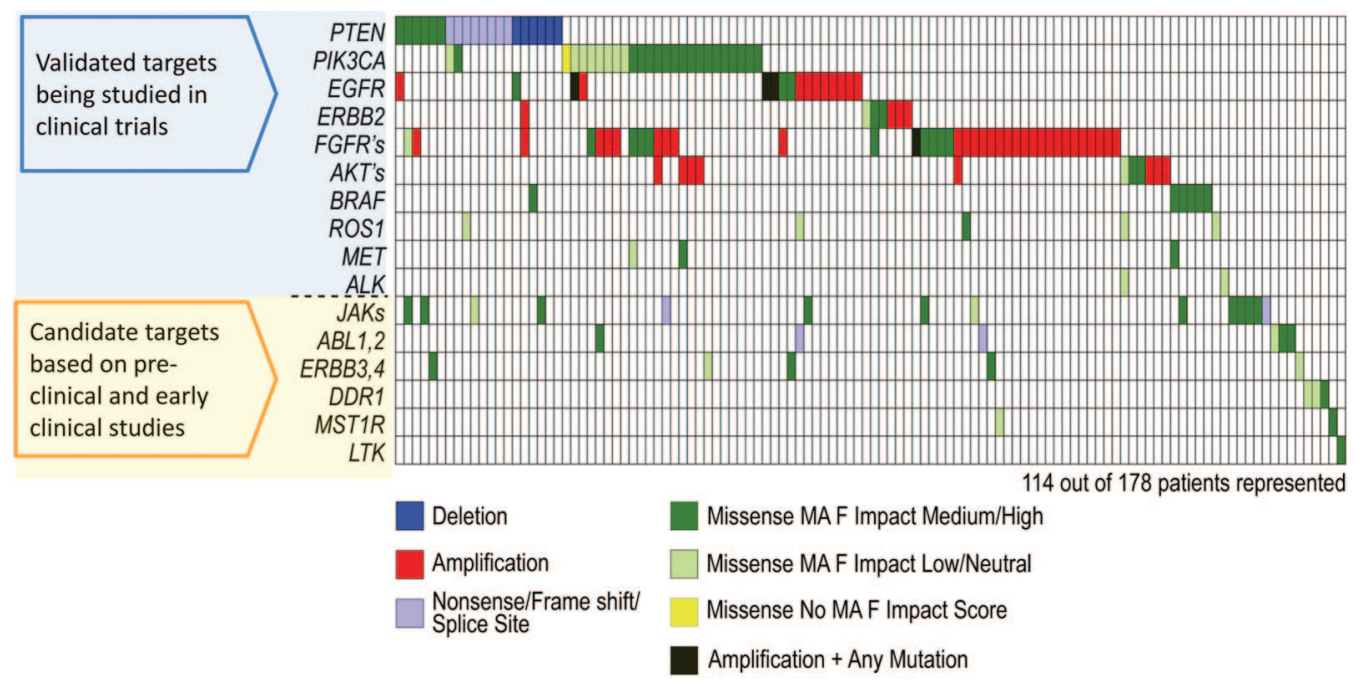

Figure 1. Druggable genetic alterations in squamous cell carcinoma: The Cancer Genome Atlas data. Reprinted from [8] with permission. Abbreviation: MA F impact: Functional impact of mutation determined by Mutation Assessor score.

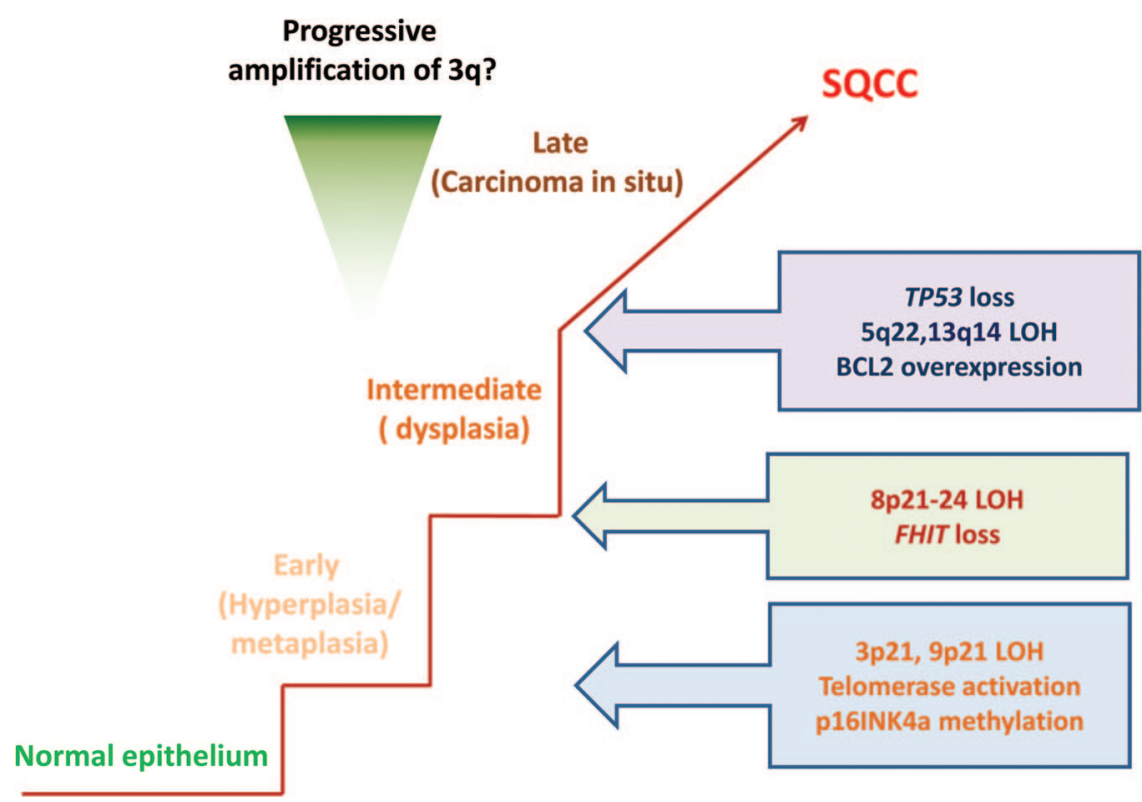

Figure 2. Schematic representation of possible genetic alterations that correlate with SQCC progression $[6,13,16,139]$. Adapted from Wistuba et al. and Drilon et al.

Abbreviation: LOH: loss of heterozygosity.

\section{Chromosomal Region $3 q$}

Evidence from previous studies, including the recently published TCGA study, strongly suggests a role for selective amplification of 3q in the pathogenesis of SQCC (Fig. 2) [8]. Genomic gain at this region has been considered to be among the most prevalent and significant molecular aberrations in SQCC [14]. Gains in this region were observed in as many as $86 \%$ of SQCC samples (19 out of 22) but only $21 \%$ of adenocarcinoma samples (3 out of 14) in a study by Kang et al. [15]. In a longitudinal bronchoscopic surveillance study, patients with premalignant bronchial dysplastic lesions were serially biopsied. None of the patients with low-grade lesions demonstrated copy number changes in the $3 q$ region, whereas all patients with high-grade lesions demonstrated $3 q$ amplification [16]. The majority of patients with high-grade lesions and $3 q$ amplification developed invasive cancer in this study. Genes of in- terest in the 3q region include SOX2, TP63, PIK3CA, and EPHB3 $[15,17]$.

\section{Chromosomal Region 3p}

Allelic losses on the $3 p$ region have been frequently reported in lung cancer $[18,19]$. Tumor suppressor genes such as RASSF1A, FUS1, VHL, and FHIT have been mapped to this region. The extent of allelic losses at this region has been observed to be greater in tumors with a squamous histology compared with adenocarcinoma, as well as in early stages of disease development $[12,19]$. Wistuba et al. analyzed the $3 p$ region for loss of heterozygosity with a panel of 28 markers; they reported allelic losses in $78 \%$ of preneoplastic (54 samples) and $96 \%$ of lung cancer samples (in a total of 97 samples with 2 SQCC cell lines and 23 SQCC samples) [12]. In another study that assessed 3p-specific epigenetic alterations, SQCC 
Comprehensive evaluation of somatic copy number alterations (SCNAs) of SQCC samples using SNP arrays revealed an average of 323 SCNAs per sample. Of these alterations, selective amplification of 50 genes was considered significant.

samples were more frequently found to be hypermethylated at this region compared with adenocarcinomas [20].

\section{Chromosome Region 9p}

Deletions involving chromosomal loci 9p21.3, 9p24.1, and $9 p 13.1$ in SQCC were reported by TCGA [8]. These loci harbor tumor suppressor genes such as CDKN2A and PTPRD [21]. Loss of heterozygosity at the locus of CDKN2A has been observed early in the development of lung cancer (Fig. 2) [11]. This gene is known to code for two critical cell cycle regulating proteins by alternate splicing: p16INK4a and p14ARF $[22,23]$. Evidence that supports the role of CDKN2A in the early pathogenesis of SQCC originates from studies that have described epigenetic inactivation of p16INK4a in SQCC, especially in smokers (current or former) [24, 25].

Belinsky et al. also reported the frequency of p16INK4a promoter hypermethylation to correlate with histological progression of SQCC (17\% in basal cell hyperplasia, $24 \%$ in squamous metaplasia, and 50\% in carcinoma in situ) [24]. Inactivation of $C D K N 2 A$ by methylation, inactivating mutations, exon $1 \beta$ skipping, or homozygous deletions was observed in $72 \%$ of the 178 SQCC samples analyzed by TCGA [8]. PTPRD is a protein tyrosine phosphatase that plays a crucial role in STAT3 signaling and is considered to be a frequent target of inactivation in several cancers, including glioblastomas, SQCC of the head and neck and lung cancer [26].

\section{Somatic Copy Number Alterations}

Comprehensive evaluation of somatic copy number alterations (SCNAs) of SQCC samples using SNP arrays revealed an average of 323 SCNAs per sample [8]. Of these alterations, selective amplification of 50 genes was considered significant. Some of the genes affected by such SCNAs and their presumed role in oncogenesis are briefly reviewed here.

\section{SOX2}

The sex determining region $Y$ (SRY)-box 2 gene (SOX2) lies on $3 q$ and is believed to be a key oncogene in this region [16]. SOX2 participates in lung organogenesis. Mice that overexpress SOX2 demonstrate abnormal branching of the lung [27]. Overexpression of SOX 2 drives cells to prematurely commit to either neuroendocrine (cGRP positive) or prebasal (deltaNp63 positive) lineages and promotes coexpression of squamous markers such as $p 63$ and keratin 6 , suggesting a role for SOX2 in squamous differentiation [28]. These observations support a role for SOX2 as a "lineage survival oncogene" in SQCC, in which lineage-specific survival and differentiation programs are exploited by the cancer cell $[29,30]$. Evidence from cell line studies indicates that SOX2 overexpression drives anchorage independent growth and invasion in cancer cells, whereas knockdown or underexpression inhibits cell growth and metastatic potential [29, 31].
SOX2 also facilitates reprogramming of differentiated cells into pluripotent cells $[33,34]$. Overexpression of SOX2 has been proposed to maintain some of the characteristics of cancer cells [28, 34]. Nearly $20 \%$ of lung SQCC samples have been found to have SOX2 amplification [29, 32]. Squamous differentiation pathway was altered in $44 \%$ and SOX 2 was activated in $21 \%$ of the samples analyzed by TCGA [8].

\section{FOXP1}

FOXP1 plays an important role in lung and esophageal development [35]. FOX family of proteins are highly conserved with well-defined roles in cancer [36]. The FOXP1 gene is located in $3 p$, a region commonly deleted in SQCC of the lung $[12,37]$. Both tumor promoting and suppressor roles have been attributed to FOXP1. Overexpression of FOXP1 in NSCLC has been associated with better survival in patients with lung cancer [38]. Messenger RNA (mRNA) levels of FOXP1 were reported to be lower in SQCC than adenocarcinoma samples at a significant level in a study conducted by Dmitriev et al. [20]. Being a lineage-associated gene and a direct target of SOX2 repression, it is possible that downregulation of FOXP1 plays an important role in dedifferentiation [28]. Inactivation of FOXP1 was reported in $4 \%$ of the samples analyzed by TCGA [8].

\section{PDGFRA}

In a study carried out by Ramos et al., amplifications of the chromosomal region $4 q 12$ were observed in $8.7 \%$ ( 5 of 57 ) of lung SQCC samples [39]. This region harbors the genes PDGFRA and KIT. PDGFRA and KIT are frequently mutated in gastrointestinal stromal tumors (GISTs) and activating mutations in KIT are considered targetable by small molecule inhibitors such as imatinib [40]. Knockdown of PDGFRA expression by small hairpin RNA resulted in decreased cell survival and growth in a NSCLC cell line ( $\mathrm{NCl}-\mathrm{H} 1703)$ overexpressing PDGFRA, when compared to a control cell line (HCC15) [39]. Amplification of PDGFRA was observed in $4 \%$ of the cases by TCGA $[8,41]$. PDGFRA is a target of several multitargeted tyrosine kinase inhibitors, including sunitinib, pazopanib, cediranib, and nintedanib, which are currently in clinical development for NSCLC.

Sunitinib, a multitargeted tyrosine kinase inhibitor, is approved by the U.S. Food and Drug Administration for the treatment of imatinib-resistant GISTs, pancreatic neuroendocrine tumors, and advanced renal cell carcinoma; currently, it is being investigated in NSCLC. Two phase II trials investigating the use of second-line sunitinib in advanced NSCLC have shown modest benefit in NSCLC. Socinski et al. studied sunitinib 50 mg daily for 4 weeks, followed by 2 weeks off, in patients with advanced NSCLC who had progressed on prior platinum therapy; the authors reported an overall response rate (ORR) of $11.1 \%$ (95\% confidence interval [CI]: 4.6\%-21.6\%), a median progression-free survival (PFS) time of 12 weeks $(95 \% \mathrm{Cl}$ : $10-$ 16.1 weeks), and a median overall survival (OS) time of 23.4 weeks ( $95 \% \mathrm{Cl}: 17.0$ to 28.3 weeks) [42]

Novello et al. investigated continuous daily sunitinib (37.5 mg daily) in patients who had progressed on previous platinum therapy and found an ORR of $2.1 \%$ (95\% Cl: $0.1 \%-11.3 \%)$, a median PFS of 11.9 weeks ( $95 \% \mathrm{Cl}: 8.6-14.1$ weeks), and median OS of 37.1 weeks (95\% Cl: 31.1-69.7 weeks) [43]. Sunitinib maintenance therapy is currently being investigated 
in a phase III trial conducted by the ALLIANCE cooperative group (CALGB 30607).

Pazopanib, a multitargeted tyrosine kinase inhibitor, is currently being studied in phase II/III second-line studies for advanced NSCLC as monotherapy (EORTC 0809, NCT01049776) and in first-line combination therapy in patients ineligible for platinum doublet therapy (NCT01179269). In a preoperative study, pazopanib produced notable reduction in tumor volume in 30 out of 35 patients [44]

A phase II/III randomized double-blinded study was performed using carboplatin and paclitaxel in combination with cediranib $(30 \mathrm{mg}$ ) or placebo in advanced NSCLC in the firstline setting. The phase II analysis revealed a higher response rate for cediranib versus placebo (38 vs. $16 \% ; p<.001$ ) and hazard ratio (HR) for PFS of 0.77 (99\% Cl: $0.56-1.08)$ [45]. However, patients in the cediranib arm had more reported toxicities, including hypertension, hypothyroidism, and gastrointestinal toxicity.

Nintedanib (BIBF 1120) is a tyrosine kinase inhibitor with activity against vascular endothelial growth factor receptors (VEGFRs), platelet-derived growth factor receptor (PDGFRs), and fibroblast growth factor receptors (FGFRs). Okamoto et al. performed a phase I study of advanced solid tumors and established a maximum tolerated dose of 200 mg b.i.d. and observed stable disease in $76.2 \%$ of patients enrolled [46]. Nintedanib was found to be tolerated well when administered in combination with pemetrexed in a phase I study [47]. One complete response was noted and $50 \%$ of patients enrolled achieved stable disease as best overall response.

In a phase II clinical trial, two doses of single-agent nintedanib (150 mg b.i.d. versus $250 \mathrm{mg}$ b.i.d.) were compared in patients with advanced NSCLC who had progressed on platinum-based chemotherapy [48]. Median PFS was 6.9 weeks and median OS time was 21.9 weeks. Outcomes were similar in both arms. Patients with an Eastern Cooperative Oncology Group performance status of 0 or 1 had an improved median PFS of 11.6 weeks and median OS of 37.7 weeks. Results from randomized trials with this agent are expected soon. Although these agents have modest activity in metastatic NSCLC, whether they will have activity in a defined subgroup of patients with activation of PDFGRA or other related genes remains to be tested.

\section{NFE2L2, KEAP1, and CUL3}

NFE2L2 encodes the NRF2 transcription factor (nuclear factor erythroid-related factor 2). NRF2 is upregulated during times of stress and facilitates production of antioxidants, drug efflux pumps, and enzymes that mediate xenobiotic detoxification $[49,50]$. Overexpression of NRF2 thus enables cancer cells to survive hostile environments and evade therapy [51, 52].

KEAP1 (Kelch-like ECH associated protein 1) negatively regulates the activity of NRF2 by facilitating its degradation [53]. Singh et al. reported on functionally important mutations in the Kelch and intervening regions of the KEAP1 protein as well as frequent biallelic inactivation of KEAP1 in the context of NSCLC [53].

CUL3 (Cullin 3), a member of the E3 ligase complex, is also responsible for mediating the degradation of NRF2 [54]. De- creased activity of KEAP1 or CUL3 can result in intracellular accumulation of NRF2 [8]. Overall, alterations in the NRF2mediated oxidative stress pathway were present in $34 \%$ of SQCC samples analyzed by TCGA. Solis et al. studied the immunohistochemical expression of NRF2 and KEAP1 in 304 NSCLC samples and correlated increased expression of NRF2 in NSCLC ( $p=.0139, \mathrm{HR}: 1.75)$ and decreased expression of KEAP1 in SQCC ( $p=.0181$, HR: 2.09) with worse overall survival [55].

\section{EPHB3}

The Eph-ephrin signaling system constitutes membranebound tyrosine kinase receptors and ligands that are capable of participating in reverse signaling [56]. Eph-ephrin signaling system mediates several key functions, such as guiding axonal growth and angiogenesis during embryogenesis. Both tumor suppressor and promoting roles have been attributed to these genes and their roles in lung and other cancers has been reviewed elaborately [56-58].

Among several members of the Eph-ephrin family, EPHB3, $E P H A 2$, and $E P H A 3$ are frequently altered in lung cancer $[8,57$, 59-64]. Overexpression of EPHB3 has been reported in NSCLC and is associated with the promotion of a metastatic phenotype in these tumors. The ability of EPHB3 to drive metastasis is believed to be independent of its kinase activity $[64,65]$. EPHB3 was amplified in $37 \%$ of SQCC samples evaluated by the TCGA $[8,41]$. Stahl et al. reported a possible role for EPHB3 expression in mediating resistance to radiotherapy [66]. EPHB3 inhibition by siRNA also was demonstrated to increase radiation sensitivity of NSCLC cells in vitro. Given that the Ephephrin family of molecules is overexpressed across a variety of cancers, several molecules that target this signaling system are currently being investigated [56-58]. Kinase inhibitors capable of interfering with the tumor promoting effects of Ephephrin signaling, peptides and antibodies capable of inhibiting Eph-ephrin interactions, and vaccines and immunotherapies capable of facilitating tumor lysis or rejection are some of the strategies that have been described to date [67-72]. Data on the safety and efficacy of these agents in lung cancer is very limited. Agents such as XL647 and dasatinib, which are multikinase inhibitors capable of targeting Eph receptors as well, have not yet shown promising results in $\operatorname{NSCLC}[68,73]$. A better understanding of Eph/ephrin kinase signaling within the cancer cell and tumor microenvironment is necessary to develop effective targeted therapies.

\section{BCL2L1}

$\mathrm{BCL} 2 \mathrm{~L} 1$ is a pro-survival, $\mathrm{BH} 3$ domain-containing protein that plays a crucial role in inhibiting cellular apoptosis [74, 75]. Amplification of $B C L 2 L 1$ was present in a small number (about $5 \%$ ) of the SQCC samples studied by TCGA [8].

\section{Mutations}

SQCC of the lung is known to carry a very high number of protein-altering mutations [76]. A total of 48,690 nonsilent mutations were reported in 18,863 genes by TCGA in 178 SQCC tumor samples [8]. This accounted for an average of 228 nonsilent mutations per sample and a mutation rate of $8.1 / \mathrm{Mb}$ (mega base) of DNA. Of these, 10 genes (TP53, CDKN2A, PTEN, PIK3CA, KEAP1, MLL2, HLA-A, NFE2L2, NOTCH1, and RB1) were considered to be significantly mutated. In another study 
that sequenced selected oncogenes in NSCLC, mutations in $D D R 2$ and FGFR2 were reported to be restricted to smokers with SQCC [77]. In the same study, EGFR, MET, and PIK3CA mutations were more prevalent in nonsmokers with SQCC compared with smokers [77]. Some of these frequently mutated genes and their role in the pathogenesis of SQCC will be briefly reviewed here.

\section{TP53, MDM2, and RB1}

TP53, a tumor suppressor gene located on chromosome 17, is perhaps the most studied gene in cancer. The $\mathrm{p} 53$ protein is instrumental in regulating the cell cycle and apoptosis. Loss of p53 expression has been described in almost all cancer types and the TCGA reported a mutation rate of approximately $90 \%$ in SQCC [8]. MDM2 is a protein that negatively regulates p53 by blocking its $\mathrm{N}$-terminal activating domain [78]. Overexpression of MDM2 has been described in multiple tumors, including small cell lung cancer, and this gene is also considered to be potentially targetable [79]. RB1, a cell cycle-regulating gene like TP53, has been implicated in the development of multiple cancers; inactivation has been reported in 7\% of SQCC samples analyzed by TCGA.

\section{TP63}

TP63 encodes two isoforms by differential splicing that take part in contrasting cell processes $[17,80]$. The TA-p63 isoform largely functions as a tumor suppressor, whereas the deltaNp63 (also called p40) isoform is pro-oncogenic. The p40 is a truncated isoform of $\mathrm{p} 63$ and facilitates dominant-negative inactivation of tumor suppressor proteins p53 and TA-p63. Pluripotent basal cells in the epidermis typically demonstrate intense staining for this isoform [81]. Because of its ability to inhibit p53, it is believed that $p 40$ aids in the maintenance of pluripotency and a constant stem cell population in basal progenitor cells [82]. The TCGA project identified p40 as the dominant isoform expressed (89\%) in SQCC compared to TA-p63.

\section{PI3KCA}

PIK3CA is one of the most frequently altered genes in cancer $[75,83,84]$. Activation of the PI3K kinase signaling system results in AKT activation and enables cancer cells to acquire multiple "hallmark" characteristics [85]. In cell line studies with immortalized airway cells, overexpression of wild-type PIK3CA was shown to promote anchorage independent growth and migration [86]. Missense mutations of the PIK3CA gene, particularly at positions 545 and 1047 (involving the helicase and kinase domains), demonstrated a similar behavior in these cells. Evidence from studies in mice also suggests a crucial metastasis-enabling role for PIK3CA [87]. PIK3CA amplification is more frequently associated with patients with squamous histology, men, and smokers $[88,89]$.

PIK3CA was one of the significantly mutated genes in the TCGA SQCC project [8]. Interestingly, 12 of the 28 SQCC samples with PIK3CA mutations in this study carried mutations at positions 545 and 1047. Further evidence to support the role of PIK3CA in SQCC also comes from studies examining $P T E N-$ a gene that regulates the PIK3CA signaling cascade and is significantly altered in these tumors [8].

\section{PTEN}

The PTEN gene is a tumor suppressor that is frequently altered in cancer [75]. PTEN catalyzes the dephosphorylation and inactivation of intracellular signaling molecule phosphatidylinositol-3,4,5-trisphosphate (PIP3), and inactivation of PTEN results in the increase in $A K T$ signaling [85]. PTEN and TP53 are often co-repressed in NSCLC [90]. Inactivation of PTEN is common in SQCC and occurs early in the disease [91]. TCGA reported PTEN alterations in $15 \%$ of the samples and these alterations were more frequently associated with the classical and primitive subtypes (based on expression profiling) [8].

Loss of PTEN can disrupt negative feedback on cell proliferation and facilitate metastasis by affecting FAK/ CDC42RhoGTPase-based pathways, which are capable of driving cell proliferation, adhesion, and migration [92, 93]. Trials evaluating agents that inhibit PIK3CA and AKT are at various stages of clinical development. A phase Il study of the PI3K inhibitor BKM 120 in pretreated metastatic NSCLC displaying PI3K pathway is currently underway (NCT01297491). A phase I/II study of BKM 120 in combination with every-3-week carboplatin and paclitaxel is also under investigation for the treatment of metastatic SQCC (NCT01820325).

\section{EGFR}

EGFR mutations were more frequent in SQCC tumor samples from nonsmokers compared with those from smokers ( $8 \%$ vs. 2.1\%) [77]. Unlike adenocarcinoma, exon 19 deletions and exon

Although these studies report an association between EGFR mutation status and better outcomes with EGFR TKIs, outcomes in EGFR TKI-treated SQCC are usually inferior in comparison to adenocarcinomas.

21 mutations are uncommon in SQCC of the lung [94]. Only a limited number of studies have examined the frequency of EGFR mutations in SQCC of the lung. EGFR mutations involving exon 19 or 21 were present in $15 \%-46 \%$ of lung SQCC samples examined in two small studies from Asia $[95,96]$. While no exon 19 deletion or L858R mutations were observed in TCGA study, two samples were found to have EGFR mutations (L861Q) known to confer sensitivity to gefitinib or erlotinib [6].

Although these studies report an association between EGFR mutation status and better outcomes with EGFR TKIs, outcomes in EGFR TKI-treated SQCC are usually inferior in comparison to adenocarcinomas $[95,97]$. A statistically nonsignificant correlation between increased survival and combination therapy with cetuximab and chemotherapy was observed in 377 patients with advanced SQCC in the FLEX (First-Line ErbituX in lung cancer) trial (HR for death: 0.8, 95\% Cl: 0.64-1.00) [98]. However, increased EGFR expression levels were considered to correlate with better outcomes in the FLEX trial [99]. A similar association between increased EGFR gene copy number and improved outcomes with EGFR-targeted therapies has also been reported in other studies [100]. Although EGFR amplifications were reported in $7 \%$ of the samples analyzed by TCGA, the extent to which these patients will respond to existing EGFR-targeted therapies is uncertain at this time $[8,99,100]$. 
The EGFR vIII mutation, commonly associated with gliomas, has also been reported in about $5 \%$ of the SQCC samples analyzed by Ji et al. [101, 102]. This variant is characterized by the deletion of exons $2-7$ that prevents ligand binding but confers constitutively active kinase activity to the receptor [103]. Decreased response to therapy with cisplatin and cetuximab was reported in head and neck SQCCs (HNSCCS) harboring this variant [104]. Evidence from a cell line study also suggests that EGFR vIll harboring tumors are relatively resistant (>40 fold more resistant compared with L858R variant) to gefitinib and erlotinib [102]. Although strategies capable of targeting this variant have been described in the literature, none are currently available for clinical use [105]. Trials investigating several existing and next-generation agents capable of inhibiting EGFR signaling are currently underway (Table 1).

\section{DDR2}

$D D R 2$ (discoidin domain containing receptor 2), a tyrosine kinase receptor that binds fibrillar collagen, was recently reported as a potentially targetable mutation in SQCC of the lung, with an estimated frequency close to 4\% [106]. Pathways downstream of $D D R 2$ regulate cell differentiation, proliferation, and migration. Hammerman et al. tested several multikinase inhibitors and found that the use of dasatinib facilitated cell death in DDR2-mutated SQCC cell lines [106]. A phase II study to evaluate the efficacy of dasatinib in advanced SQCC of the lung (NCT01491633) was recently closed because of toxicity issues. Novel DDR2 inhibitors with better safety profiles are urgently required.

\section{FGFR1}

FGFR1 is a tyrosine receptor kinase that specifically binds ligands of the fibroblast growth factor family and is responsible for inducing mitosis, cell differentiation, and angiogenesis [107]. Preclinical studies have demonstrated FGFR1 amplification predominantly involving SQCC of the lung in current smokers [108]. Overexpression of FGFR1 has been reported in $9.7 \%-21 \%$ of SQCC samples $[108,109]$. Activation of FGFR1 was present in $7 \%$ of the samples analyzed by TCGA [8]. Weiss et al. described the use of a small molecule PD173074 that nonspecifically blocks the FGF family of receptors. In their study, PD173074 was able to inhibit tumor growth and promote apoptosis in cells overexpressing FGFR1. These findings suggest that the blockade of FGFR1 may be a promising target in treating SQCC. Multiple FGFR inhibitors are in early clinical development [107]. BGJ398 and AZD4546 are pan-FGFR kinase inhibitors currently being studied in the phase I setting. Multikinase inhibitors targeting FGFR in combination with VEGFR and PDGFR are also in early phases of clinical testing, including E-3810, a dual VEGFR-FGFR tyrosine kinase inhibitor [110].

\section{ERBB2}

$E R B B 2$ is a proto-oncogene located on the long arm of chromosome 17 (17q12) and encodes HER2/Neu, a receptor tyrosine kinase of the epidermal growth factor receptor family. Overexpression of HER2 contributes to the pathogenesis of many cancers including breast, ovarian, gastric, and salivary gland cancers [111-114]. Stephens et al. reported mutations in ERBB2 kinase domain in $4 \%$ of the tumor samples from pa- tients with lung cancer [115]. All patients with ERBB2 mutations had adenocarcinoma histology. Evidence suggests a role for ERBB2 signaling in mediating resistance of lung cancers to EGFR-targeting therapies [116]. Given that mutation and amplification of ERBB2 in SQCC were reported in $3 \%$ and $2 \%$ of the samples analyzed by TCGA, respectively, therapy targeting ERBB2 could potentially benefit a subset of patients with SQCC $[8,41]$.

\section{NF1}

The neurofibromin 1 (NF1) gene is located on the chromosomal region $17 q$. This gene plays an important role in the development of epidermal cells [117]. It is often expressed in basal keratinocytes during the fetal period. NF1 plays the role of a tumor suppressor protein by inhibiting RAS. Mutations in NF1 have been reported in both NSCLC and small cell lung cancer $[61,118,119]$. It is possible that $N F 1$ plays an important role in the pathogenesis of SQCC given that it is required for the normal development of epidermal cells. NF1 was inactivated in $11 \%$ of the samples examined by TCGA [8]. More studies are definitely required to establish the role of NF1 in SQCC.

\section{NOTCH1}

NOTCH signaling pathways are important for embryogenesis and controlling cell differentiation. Mutations in the NOTCH signaling pathway have been described in the development of many cancers [120]. Activating mutations in the $\mathrm{NOTCH} 1$ gene have been implicated in more than $50 \%$ of all cases of T-cell acute lymphoblastic leukemia [121]. Inhibition of NOTCH signaling has antiproliferative effects in mouse models [122]. Loss of function in the NOTCH signaling pathway secondary to truncating mutations of NOTCH1 have been described in HNSCC [123]. TCGA investigators reported NOTCH1 mutations in $8 \%$ of SQCCs of the lung [8]. Of these mutations, 8 of 17 were found to be truncating mutations similar to those described in SQCC of the head and neck.

\section{HLA-A}

Human leukocyte antigen A $(H L A-A)$ is encoded by the $H L A-A$ locus on chromosome $6 \mathrm{p}$. HLA-A is part of the major histocompatibility complex class I, which functions as part of the immune system to present foreign polypeptides to the immune system and specifically target cells for destruction by activation of T lymphocytes [124]. Loss of function mutations within the HLA-A gene locus in SQCC, primarily through nonsense or splicing mutations, were reported for the first time by TCGA [8]. These mutations in the HLA-A gene suggest a possible mechanism by which tumor cells can successfully evade immune destruction and present a potential role for immunotherapy.

\section{MLL2}

Mixed lineage leukemia 2 ( $M L L 2$ ) is a member of the trithorax family of human genes, encoding a histone-lysine- $\mathrm{N}$ methyltransferase on chromosome 12q13.12 [125]. MLL2 is involved in epigenetic programming and embryonic development and is overexpressed in several malignancies such as medulloblastoma, breast cancer, colon cancer, and non-Hodgkin's lymphoma [126-128]. MLL2 was reported as significantly mutated in SQCC of the lung with 7 silent mutations, 40 nonsilent mutations, and 18 frame shift and 
Table 1. Some of the actively recruiting clinical trials testing targeted agents in squamous cell carcinoma of the lung

\begin{tabular}{lll}
\hline Target & Drug & Clinicaltrials.gov numbers \\
\hline EGFR & $\begin{array}{l}\text { Erlotinib, panitumumab, gefitinib, afatinib, } \\
\text { cetuximab, RO5083945 }\end{array}$ & $\begin{array}{l}\text { NCT00979212, NCT00661193, NCT01294306, NCT01059188, } \\
\text { NCT00966472, NCT01013831, NCT00835471, NCT010204697, } \\
\text { NCT01702714, NCT01485809, NCT01523587, NCT00946712 }\end{array}$ \\
Angiogenesis & $\begin{array}{l}\text { Bevacizumab, ramucirumab, NGR-hTNF, } \\
\text { nintedanib }\end{array}$ & NCT00946712, NCT01160744, NCT00994097, NCT01346540 \\
AKT & MK2206 & NCT01294306 \\
PI3K & PX866, BKM120 & NCT01204099, NCT01297491 \\
ERBB & Dacomitinib (pan-ErbB), M121 (ErbB3) & NCT01441128, NCT01451632 \\
Other & Dasatinib (DDR2), crizotinib (EML4-ALK) & NCT01491633, NCT01514864, NCT01441128
\end{tabular}

Data is from the ClinicalTrials.gov database, accessed November 5, 2012.

nonsense mutations by TCGA [8]. Several emerging therapeutic strategies targeting epigenetic pathways are currently being investigated $[129,130]$.

\section{MRNA EXPRESSION PROFILING}

Using expression arrays, Wilkerson et al. identified four mRNA expression subtypes of SQCC of the lung: classical, primitive, basal, and secretory [131]. These findings were replicated in the SQCC TCGA study [8]. The classical subtype was the most prevalent ( $36 \%$ of TCGA samples) and was predominantly encountered in men, smokers, and tumors overexpressing genes responsible for xenobiotic metabolism and those on $3 q$ including SOX2, TP63, and PIK3CA. The primitive type, accounting for $15 \%$ of all TCGA samples, was characterized by rapid cell proliferation, poor differentiation, and worse overall survival. The basal subtype (25\%), in contrast to the primitive type, was characterized by a well-differentiated phenotype. Genes altered in basal tumors played an important role in cell adhesion and epidermal development. Lastly, the secretory type (24\%) was associated with alteration in genes responsible for mediating immune responses as well as mucin and surfactant production. The clinical implications of these findings need to be explored in larger groups of patients with SQCC of the lung.

\section{STRUCTURAL VARIATIONS}

Translocations involving $A L K, R E T$, and ROS1 have been reported in NSCLC (mostly adenocarcinoma) and are amenable for therapy with targeted agents such as crizotinib [132-135]. On average, 165 somatic rearrangements per sample were identified in the tumor samples in the SQCC TCGA project [8]. None of these events were recurrent or associated with a fusion protein. Additional work needs to be done to understand the significance of these findings.

\section{EPIGENETIC Alterations}

Epigenetic alteration by differential methylation of genes is common in SQCC [136]. Hypermethylated differentially methylated regions (DMRs) were overrepresented in SQCC samples on genome-wide DNA methylation analysis of NSCLC samples [136]. Of these, 287 DMRs were found to be unique to SQCC, whereas only 26 DMRs were unique for adenocarcinomas. Although there was heterogeneity in the functions of these hypermethylated genes, genes associated with the regulation of transcription, cytoskeletal organization, and cell cycle were overrepresented in these samples. Promoter hypermethylation was more frequently reported in the classical subtype by TCGA investigators [8, 131]. Hypermethylation of the p16INK4a promoter (especially in smokers) has been discussed previously. Epigenetic changes that bring about the inactivation of TP53 have also been reported in SQCC [137]. The HIC1-SIRT1-p53 circular loop deregulation has been described as one way of achieving such an inactivation. Decreased expression of the hypermethylated in cancer (HIC1) gene renders it incapable of repressing SIRT1, a deacetylase that inactivates TP53. Differential methylation patterns of a gene cluster on 9p21 composed of p16INK4a, p15INK4b, and p14ARF promoters between central and peripheral SQCC has also been reported [138]. The significance of these differences is unclear.

\section{SUMMARY}

Ongoing efforts by TCGA investigators will map the genomic landscape of 500 tumor samples from patients with SQCC of the lung by the end of this year. These and other large-scale efforts will help us understand the molecular pathogenesis of SQCC of the lung better over the next few years. Carefully designed preclinical and clinical studies to translate these findings in the clinic will very likely improve the outcomes of patients with SQCC of the lung.

\section{ACKNOWLEDGMENTS}

Melissa Rooney and Siddhartha Devarakonda contributed equally to this work.

\section{Author Contributions}

Conception/Design: Ramaswamy Govindan

Collection and/or assembly of data: Melissa Rooney, Siddhartha Devarakonda Data analysis and interpretation: Melissa Rooney, Siddhartha Devarakonda, Ramaswamy Govindan

Manuscript writing: Melissa Rooney, Siddhartha Devarakonda, Ramaswamy Govindan

Final approval of manuscript: Melissa Rooney, Siddhartha Devarakonda, Ramaswamy Govindan

\section{DISCLOSURES}

Ramaswamy Govindan: Bristol-Myers Squibb, Merck, BoehringerIngelheim, Covidien, Abbott Oncology (C/A). The other authors indicated no financial relationships.

Section editors: Lecia Sequist: GSK, Clovis Oncology (C/A); Nate Pennell: Oncogenex, Teva (C/A); Pfizer, Genentech, Imclone, Sanofi, Helsinn, CanBas (RF); Natasha Leighl: None

Reviewer "A": None

C/A: Consulting/advisory relationship; RF: Research funding; E: Employment; $\mathrm{H}$ : Honoraria received; OI: Ownership interests; IP: Intellectual property rights/inventor/patent holder; SAB: scientific advisory board 


\section{REFERENCES}

1. Howlader NNA, Krapcho M, Neyman N et al. SEER cancer statistics review, 1975-2009 (vintage 2009 populations). Bethesda, MD: National Cancer Institute, 2012.

2. World Health Organization. Cancer Fact Sheet Number 297. Geneva, Switzerland: World Health Organization, 2012.

3. Siegel R, Naishadham D, Jemal A. Cancer statistics, 2013. CA Cancer J Clin 2013;63:11-30.

4. Navada S, Lai P, Schwartz A et al. Temporal trends in small cell lung cancer: Analysis of the national Surveillance Epidemiology and End-Results (SEER) database. J Clin Oncol 2006;24(suppl 18):384S.

5. Molina JR, Yang P, Cassivi SD et al. Non-small cell lung cancer: Epidemiology, risk factors, treatment, and survivorship. Mayo Clin Proc 2008;83:584594.

6. Travis WD. Pathology of lung cancer. Clin Chest Med 2011;32:669-692.

7. DeVita VT, Lawrence TS, Rosenberg SA. Cancer: Principles and practice of oncology. Philadelphia, PA: Lippincott Williams \& Wilkins, 2011.

8. Hammerman PS, Hayes DN, Wilkerson MD et al. Comprehensive genomic characterization of squamous cell lung cancers. Nature 2012;489:519-525.

9. Knudson AG. Mutation and cancer: Statistical study of retinoblastoma. Proc Natl Acad Sci U S A 1971;68:820-823.

10. Berger AH, Knudson AG, Pandolfi PP. A continuum model for tumour suppression. Nature 2011; 476:163-169.

11. Wistuba II, Behrens C, Milchgrub S et al. Sequential molecular abnormalities are involved in the multistage development of squamous cell lung carcinoma. Oncogene 1999;18:643-650.

12. Wistuba II, Behrens C, Virmani AK et al. High resolution chromosome $3 p$ allelotyping of human lung cancer and preneoplastic/preinvasive bronchial epithelium reveals multiple, discontinuous sites of $3 p$ allele loss and three regions of frequent breakpoints. Cancer Res 2000;60:19491960.

13. Drilon A, Rekhtman N, Ladanyi M et al. Squamous-cell carcinomas of the lung: Emerging biology, controversies, and the promise of targeted therapy. Lancet Oncol 2012;13:e418-e426.

14. Qian J, Massion PP. Role of chromosome 3q amplification in lung cancer. J Thorac Oncol 2008;3: 212-215.

15. Kang JU, Koo SH, Kwon KC et al. Identification of novel candidate target genes, including EPHB3, MASP1 and SST at 3q262-q29 in squamous cell carcinoma of the lung. BMC Cancer 2009;9:237.

16. McCaughan F, Pole JC, Bankier AT et al. Progressive $3 q$ amplification consistently targets SOX2 in preinvasive squamous lung cancer. Am J Respir Crit Care Med 2010;182:83-91.

17. Yang A, McKeon F. P63 and P73: P53 mimics, menaces and more. Nat Rev Mol Cell Biol 2000;1: 199-207.

18. Zabarovsky ER, Lerman MI, Minna JD. Tumor suppressor genes on chromosome $3 p$ involved in the pathogenesis of lung and other cancers. Oncogene 2002;21:6915-6935.

19. Herbst RS, Heymach JV, Lippman SM. Lung cancer. N Engl J Med 2008;359:1367-1380.

20. Dmitriev AA, Kashuba VI, Haraldson Ket al. Ge- netic and epigenetic analysis of non-small cell lung cancer with Notl-microarrays. Epigenetics 2012;7: 502-513.

21. Julien SG, Dubé N, Hardy S et al. Inside the human cancer tyrosine phosphatome. Nat Rev Cancer 2011;11:35-49.

22. Liggett WH, Sidransky D. Role of the p16 tumor suppressor gene in cancer. J Clin Oncol 1998;16: 1197-1206.

23. Sherr CJ. Divorcing ARF and p53: An unsettled case. Nat Rev Cancer 2006;6:663-673.

24. Belinsky SA, Nikula KJ, Palmisano WA et al. Aberrant methylation of $\mathrm{p} 16$ (INK4a) is an early event in lung cancer and a potential biomarker for early diagnosis. Proc Natl Acad Sci U S A 1998;95:1189111896.

25. Belinsky SA, Palmisano WA, Gilliland FD et al. Aberrant promoter methylation in bronchial epithelium and sputum from current and former smokers. Cancer Res 2002;62:2370-2377.

26. Veeriah S, Brennan C, Meng S et al. The tyrosine phosphatase PTPRD is a tumor suppressor that is frequently inactivated and mutated in glioblastoma and other human cancers. Proc Natl Acad Sci U S A 2009;106:9435-9440.

27. Gontan C, de Munck A, Vermeij M et al. Sox 2 is important for two crucial processes in lung development: Branching morphogenesis and epithelial cell differentiation. Dev Biol 2008;317:296-309.

28. Hussenet T, du Manoir S. SOX2 in squamous cell carcinoma: Amplifying a pleiotropic oncogene along carcinogenesis. Cell Cycle 2010;9:14801486.

29. Bass AJ, Watanabe $\mathrm{H}$, Mermel $\mathrm{CH}$ et al. SOX2 is an amplified lineage-survival oncogene in lung and esophageal squamous cell carcinomas. Nature Gen 2009;41:1238-1242.

30. Garraway LA, Sellers WR. Lineage dependency and lineage-survival oncogenes in human cancer. Nat Rev Cancer 2006;6:593-602.

31. Xiang R, Liao D, Cheng T et al. Downregulation of transcription factor SOX2 in cancer stem cells suppresses growth and metastasis of lung cancer. Br J Cancer 2011;104:1410-1417.

32. Hussenet T, Dali S, Exinger J et al. SOX2 is an oncogene activated by recurrent 3q26.3 amplifications in human lung squamous cell carcinomas. PLoS One 2010;5:e8960.

33. Takahashi K, Tanabe K, Ohnuki M et al. Induction of pluripotent stem cells from adult human fibroblasts by defined factors. Cell 2007;131:861872.

34. Boyer LA, Lee TI, Cole MF et al. Core transcriptional regulatory circuitry in human embryonic stem cells. Cell 2005;122:947-956.

35. Shu W, Lu MM, Zhang Y et al. Foxp2 and Foxp1 cooperatively regulate lung and esophagus development. Development 2007;134:1991-2000.

36. Myatt SS, Lam EW. The emerging roles of forkhead box (Fox) proteins in cancer. Nat Rev Cancer 2007;7:847-859.

37. Banham AH, Beasley N, Campo E et al. The FOXP1 winged helix transcription factor is a novel candidate tumor suppressor gene on chromosome 3p. Cancer Res 2001;61:8820-8829.

38. Feng J, Zhang $X$, Zhu $\mathrm{H}$ et al. High expression of FoxP1 is associated with improved survival in pa- tients with non-small cell lung cancer. Am J Clin Pathol 2012;138:230-235.

39. Ramos AH, Dutt A, Mermel C et al. Amplification of chromosomal segment $4 q 12$ in non-small cell lung cancer. Cancer Biol Ther 2009;8:20422050.

40. Heinrich $M C$, Corless $C L$, Duensing $A$ et al. PDGFRA activating mutations in gastrointestinal stromal tumors. Science 2003;299:708-710.

41. Cerami E, Gao J, Dogrusoz U et al. The cBio cancer genomics portal: An open platform for exploring multidimensional cancer genomics data. Cancer Discov 2012;2:401-404

42. Socinski MA, Novello S, Brahmer JR et al. Multicenter, phase II trial of sunitinib in previously treated, advanced non-small-cell lung cancer. J Clin Oncol 2008;26:650-656.

43. Novello S, Scagliotti GV, Rosell R et al. Phase II study of continuous daily sunitinib dosing in patients with previously treated advanced non-small cell lung cancer. Br J Cancer 2009;101:1543-1548.

44. Altorki N, Lane ME, Bauer T et al. Phase II proof-of-concept study of pazopanib monotherapy in treatment-naive patients with stage I/II resectable non-small-cell lung cancer. J Clin Oncol 2010; 28:3131-3137.

45. Goss GD, Arnold A, Shepherd FA et al. Randomized, double-blind trial of carboplatin and paclitaxe with either daily oral cediranib or placebo in advanced non-small-cell lung cancer: NCIC clinical trials group BR24 study. J Clin Oncol 2010;28:49-55.

46. Okamoto I, Kaneda H, Satoh T et al. Phase I safety, pharmacokinetic, and biomarker study of BIBF 1120, an oral triple tyrosine kinase inhibitor in patients with advanced solid tumors. Mol Cancer Ther 2010;9:2825-2833.

47. Ellis PM, Kaiser R, Zhao Y et al. Phase I openlabel study of continuous treatment with BIBF 1120, a triple angiokinase inhibitor, and pemetrexed in pretreated non-small cell lung cancer patients. Clin Cancer Res 2010;16:2881-2889.

48. Reck M, Kaiser R, Eschbach C et al. A phase I double-blind study to investigate efficacy and safety of two doses of the triple angiokinase inhibitor BIBF 1120 in patients with relapsed advanced non-small-cell lung cancer. Ann Oncol 2011;22: 1374-1381.

49. Ishii T, Itoh K, Takahashi S et al. Transcription factor Nrf2 coordinately regulates a group of oxidative stress-inducible genes in macrophages. J Biol Chem 2000;275:16023-16029.

50. Itoh K, Chiba T, Takahashi S et al. An Nrf2/small Maf heterodimer mediates the induction of phase I detoxifying enzyme genes through antioxidant response elements. Biochem Biophys Res Commun 1997;236:313-322.

51. Wang XJ, Sun Z, Villeneuve NF et al. Nrf2 enhances resistance of cancer cells to chemotherapeutic drugs, the dark side of Nrf2. Carcinogenesis 2008;29:1235-1243.

52. Singh A, Bodas M, Wakabayashi N et al. Gain of Nrf2 function in non-small-cell lung cancer cells confers radioresistance. Antioxid Redox Signal 2010;13:1627-1637.

53. Singh A, Misra V, Thimmulappa RK et al. Dysfunctional KEAP1-NRF2 interaction in non-smallcell lung cancer. PLoS Med 2006;3:e420.

54. Kobayashi A, Kang MI, Okawa Het al. Oxidative stress sensor Keap1 functions as an adaptor for 
Cul3-based E3 ligase to regulate proteasomal degradation of Nrf2. Mol Cell Biol 2004;24:7130-7139.

55. Solis LM, Behrens C, Dong W et al. Nrf2 and Keap1 abnormalities in non-small cell lung carcinoma and association with clinicopathologic features. Clin Cancer Res 2010;16:3743-3753.

56. Pasquale EB. Eph receptors and ephrins in cancer: Bidirectional signalling and beyond. Nat Rev Cancer 2010;10:165-80.

57. Li JJ, Xie D. The roles and therapeutic potentials of Ephs and ephrins in lung cancer. Exp Cell Res 2013;319:152-159.

58. Nievergall E, Saunders T, Lackmann M. Targeting of EPH receptor tyrosine kinases for anticancer therapy. Crit Rev Oncog 2012;17:211-232.

59. Davies H, Hunter C, Smith R et al. Somatic mutations of the protein kinase gene family in human lung cancer. Cancer Res 2005;65:7591-7595.

60. Greenman C, Stephens P, Smith R et al. Patterns of somatic mutation in human cancer genomes. Nature 2007;446:153-158.

61. Ding L, Getz G, Wheeler DA et al. Somatic mutations affect key pathways in lung adenocarcinoma. Nature 2008;455:1069-1075.

62. Wood LD, Calhoun ES, Silliman N et al. Somatic mutations of GUCY2F, EPHA3, and NTRK3 in human cancers Hum Mutat 2006;27:1060-1061.

63. Faoro L, Singleton PA, Cervantes GM et al. EphA2 mutation in lung squamous cell carcinoma promotes increased cell survival, cell invasion, focal adhesions, and mammalian target of rapamycin activation. J Biol Chem 2010;285:18575-18585.

64. Ji XD, Li G, Feng YX et al. EphB3 is overexpressed in non-small-cell lung cancer and promotes tumor metastasis by enhancing cell survival and migration. Cancer Res 2011;71:1156-1166.

65. Li G, Ji XD, Gao H et al. EphB3 suppresses nonsmall-cell lung cancer metastasis via a PP2A/ RACK1/Akt signalling complex. Nat Commun 2012; 3:667.

66. Ståhl S, Kaminskyy VO, Efazat G et al. Inhibition of Ephrin B3-mediated survival signaling contributes to increased cell death response of non-small cell lung carcinoma cells after combined treatment with ionizing radiation and PKC 412. Cell Death Dis 2013;4:e454.

67. Pietanza MC, Gadgeel SM, Dowlati A et al. Phase II study of the multitargeted tyrosine kinase inhibitor XL647 in patients with non-small-cell lung cancer. J Thorac Oncol 2012;7:856-865.

68. Johnson FM, Bekele BN, Feng L et al. Phase II study of dasatinib in patients with advanced nonsmall-cell lung cancer. J Clin Oncol 2010;28:46094615.

69. Xu Z, Jin H, Qian Q. Humanized anti-EphB4 antibodies for the treatment of carcinomas and vasculogenesis-related diseases. Expert Opin Ther Pat 2009;19:1035-1037.

70. Chrencik JE, Brooun A, Kraus ML et al. Structural and biophysical characterization of the EphB4*ephrinB2 protein-protein interaction and receptor specificity. J Biol Chem 2006;281:2818528192.

71. Hatano M, Eguchi J, Tatsumi T et al. EphA2 as a glioma-associated antigen: A novel target for glioma vaccines. Neoplasia 2005;7:717-722.

72. Hammond SA, Lutterbuese R, Roff S et al. Selective targeting and potent control of tumor growth using an EphA2/CD3-Bispecific single-chain antibody construct. Cancer Res 2007;67:39273935.

73. Pietanza MC, Lynch TJ, Lara PN et al. XL647-a multitargeted tyrosine kinase inhibitor: Results of a phase II study in subjects with non-small cell lung cancer who have progressed after responding to treatment with either gefitinib or erlotinib. J Thorac Oncol 2012;7:219-226.

74. Lessene G, Czabotar PE, Colman PM. BCL-2 family antagonists for cancer therapy. Nat Rev Drug Discov 2008;7:989-1000.

75. Hanahan D, Weinberg RA. Hallmarks of cancer: The next generation. Cell 2011;144:646-674.

76. Kan Z, Jaiswal BS, Stinson J et al. Diverse somatic mutation patterns and pathway alterations in human cancers. Nature 2010;466:869-873.

77. An SJ, Chen ZH, Su J et al. Identification of enriched driver gene alterations in subgroups of nonsmall cell lung cancer patients based on histology and smoking status. PLoS One 2012;7:e40109.

78. Kubbutat MH, Jones SN, Vousden KH. Regulation of p53 stability by Mdm2. Nature 1997;387: 299-303.

79. Wade M, Li YC, Wahl GM. MDM2, MDMX and p53 in oncogenesis and cancer therapy. Nat Rev Cancer 2012;13:83-96.

80. Yang A, Kaghad M, Wang Y et al. p63, a p53 homolog at 3q27-29, encodes multiple products with transactivating, death-inducing, and dominantnegative activities. Mol Cell 1998;2:305-316.

81. Parsa R, Yang A, McKeon F et al. Association of p63 with proliferative potential in normal and neoplastic human keratinocytes. J Invest Dermatol 1999;113:1099-1105.

82. Yang A, Schweitzer R, Sun D et al. p63 is essential for regenerative proliferation in limb, craniofacial and epithelial development. Nature 1999;398: 714-718.

83. Samuels $Y$, Wang $Z$, Bardelli A et al. High frequency of mutations of the PIK3CA gene in human cancers. Science 2004;304:554.

84. Engelman JA. Targeting PI3K signalling in cancer: Opportunities, challenges and limitations. Nat Rev Cancer 2009;9:550-562.

85. Vivanco I, Sawyers CL. The phosphatidylinositol 3-Kinase AKT pathway in human cancer. Nat Rev Cancer 2002;2:489-501.

86. Okudela K, Suzuki M, Kageyama S et al. PIK3CA mutation and amplification in human lung cancer. Pathol Int 2007;57:664-671.

87. Samuels Y, Diaz LA, Schmidt-Kittler O et al. Mutant PIK3CA promotes cell growth and invasion of human cancer cells. Cancer Cell 2005;7:561-573.

88. Kawano O, Sasaki H, Okuda K et al. PIK3CA gene amplification in Japanese non-small cell lung cancer. Lung Cancer 2007;58:159-160.

89. Yamamoto H, Shigematsu H, Nomura $M$ et al. PIK3CA mutations and copy number gains in human lung cancers. Cancer Res 2008;68:6913-6921.

90. Andjelkovic T, Bankovic J, Stojsic J et al. Coalterations of $\mathrm{p} 53$ and PTEN tumor suppressor genes in non-small cell lung carcinoma patients. Transl Res 2011;157:19-28.

91. Marsit CJ, Zheng S, Aldape Ket al. PTEN expression in non-small-cell lung cancer: Evaluating its relation to tumor characteristics, allelic loss, and epigenetic alteration. Hum Pathol 2005;36:768776.

92. Liliental J, Moon SY, Lesche R et al. Genetic deletion of the Pten tumor suppressor gene promotes cell motility by activation of Rac1 and Cdc42 GTPases. Curr Biol 2000;10:401-404.

93. Hood JD, Cheresh DA. Role of integrins in cell invasion and migration. Nat Rev Cancer 2002;2:91100.

94. Sharma SV, Bell DW, Settleman J et al. Epidermal growth factor receptor mutations in lung cancer. Nat Rev Cancer 2007;7:169-181.

95. Duan JC, An TT, Wu MN et al. [Correlation between the efficacy of epidermal growth factor receptor tyrosine kinase inhibitors and EGFR mutations in advanced squamous cell lung cancer]. Zhonghua Jie He He Hu Xi Za Zhi 2012;35:323-328.

96. Park SH, Ha SY, Lee JI et al. Epidermal growth factor receptor mutations and the clinical outcome in male smokers with squamous cell carcinoma of lung. J Korean Med Sci 2009;24:448-452.

97. Shukuya T, Takahashi T, Kaira R et al. Efficacy of gefitinib for non-adenocarcinoma non-small-cell lung cancer patients harboring epidermal growth factor receptor mutations: A pooled analysis of published reports. Cancer Sci 2011;102:10321037.

98. Pirker R, Pereira JR, Szczesna A et al. Cetuximab plus chemotherapy in patients with advanced non-small-cell lung cancer (FLEX): An open-label randomised phase III trial. Lancet 2009;373:15251531.

99. Pirker R, Pereira JR, von Pawel J et al. EGFR expression as a predictor of survival for first-line chemotherapy plus cetuximab in patients with advanced non-small-cell lung cancer: Analysis of data from the phase 3 FLEX study. Lancet Oncol 2012;13:33-42.

100. Hirsch FR, Varella-Garcia M, Cappuzzo F et al. Combination of EGFR gene copy number and protein expression predicts outcome for advanced non-small-cell lung cancer patients treated with gefitinib. Ann Oncol 2007;18:752-760.

101. Nishikawa R, Ji XD, Harmon RC et al. A mutant epidermal growth factor receptor common in human glioma confers enhanced tumorigenicity. Proc Natl Acad Sci U S A 1994;91:7727-7731.

102. Ji H, Zhao X, Yuza $Y$ et al. Epidermal growth factor receptor variant III mutations in lung tumorigenesis and sensitivity to tyrosine kinase inhibitors. Proc Natl Acad Sci U S A 2006;103:7817-7822.

103. Okamoto I, Kenyon LC, Emlet DR et al. Expression of constitutively activated EGFRvIII in nonsmall cell lung cancer. Cancer Sci 2003;94:50-56.

104. Sok JC, Coppelli FM, Thomas SM et al. Mutant epidermal growth factor receptor (EGFRvIII) contributes to head and neck cancer growth and resistance to EGFR targeting. Clin Cancer Res 2006;12: 5064-5073.

105. Omidfar K, ShirvaniZ. Single domain antibodies: A new concept for epidermal growth factor receptor and EGFRvIII targeting. DNA Cell Biol 2012; 31:1015-1026.

106. Hammerman PS, Sos ML, Ramos AH et al. Mutations in the DDR2 kinase gene identify a nove therapeutic target in squamous cell lung cancer. Cancer Discov 2011;1:78-89.

107. Semrad TJ, Mack PC. Fibroblast growth factor signaling in non-small-cell lung cancer. Clin Lung Cancer 2012;13:90-95.

108. Weiss J, Sos ML, Seidel D et al. Frequent and focal FGFR1 amplification associates with therapeutically tractable FGFR1 dependency in squamous cell lung cancer. Sci Transl Med 2010;2: 62 ra93. 
109. Dutt $A$, Ramos AH, Hammerman PS et al. Inhibitor-sensitive FGFR1 amplification in human nonsmall cell lung cancer. PloS One 2011;6:e20351.

110. Perez-Moreno $P$, Brambilla E, Thomas R et al. Squamous cell carcinoma of the lung: Molecular subtypes and therapeutic opportunities. Clin Cancer Res 2012;18:2443-2451.

111. Slamon DJ, Clark GM, Wong SG et al. Human breast cancer: Correlation of relapse and survival with amplification of the HER-2/neu oncogene. Science 1987;235:177-182.

112. Vermeij J, Teugels E, Bourgain C et al. Genomic activation of the EGFR and HER2-neu genes in a significant proportion of invasive epithelial ovarian cancers. BMC Cancer 2008;8:3.

113. Jaehne J, Urmacher C, Thaler HT et al. Expression of Her2/neu oncogene product p185 in correlation to clinicopathological and prognostic factors of gastric carcinoma. J Cancer Res Clin Oncol 1992; 118:474-479.

114. Cornolti G, Ungari M, Morassi ML et al. Amplification and overexpression of HER2/neu gene and HER2/neu protein in salivary duct carcinoma of the parotid gland. Arch Otolaryngol 2007;133:1031-1036.

115. Stephens $P$, Hunter $C$, Bignell $G$ et al. Lung cancer: Intragenic ERBB2 kinase mutations in tumours. Nature 2004;431:525-526.

116. Yonesaka K, Zejnullahu K, Okamoto I et al. Activation of ERBB2 signaling causes resistance to the EGFR-directed therapeutic antibody cetuximab. Science Transl Med 2011;3:99ra86.

117. Malminen M, Peltonen S, Koivunen J et al. Functional expression of NF1 tumor suppressor protein: Association with keratin intermediate filaments during the early development of human epidermis. BMC Dermatol 2002;2:10

118. Hermonen J, Hirvonen O, Ylä-Outinen $\mathrm{H}$ et al. Neurofibromin: Expression by normal human kera- tinocytes in vivo and in vitro and in epidermal malignancies. Lab Invest 1995;73:221-228.

119. Furukawa K, Yanai N, Fujita M et al. Novel mutations of neurofibromatosis type 1 gene in smal cell lung cancers. Surg Today 2003;33:323-327.

120. Ranganathan $P$, Weaver KL, Capobianco AJ. Notch signalling in solid tumours: A little bit of everything but not all the time. Nature Rev Cancer 2011;11:338-351.

121. Weng AP, Ferrando AA, Lee $W$ et al. Activating mutations of NOTCH1 in human T cell acute lymphoblastic leukemia. Science 2004;306:269-271.

122. Moellering RE, Cornejo M, Davis TN et al. Direct inhibition of the NOTCH transcription factor complex. Nature 2009;462:182-188.

123. Agrawal N, Frederick MJ, Pickering CR et al. Exome sequencing of head and neck squamous cell carcinoma reveals inactivating mutations in NOTCH1. Science 2011;333:1154-1157.

124. Delves PJ, Roitt IM. The immune system. First of two parts. N Engl J Med 2000;343:37-49.

125. FitzGerald KT, Diaz MO. MLL2: A new mammalian member of the trx/MLL family of genes. Genomics 1999;59:187-192.

126. Parsons DW, Li M, Zhang X et al. The genetic landscape of the childhood cancer medulloblastoma. Science 2011;331:435-439.

127. Natarajan TG, Kallakury BV, Sheehan CE et al. Epigenetic regulator MLL2 shows altered expression in cancer cell lines and tumors from human breast and colon. Cancer Cell Int 2010;10:13.

128. Morin RD, Mendez-Lago M, Mungall AJ et al. Frequent mutation of histone-modifying genes in non-Hodgkin lymphoma. Nature 2011;476:298-303.

129. Popovic R, Licht JD. Emerging epigenetic targets and therapies in cancer medicine. Cancer Discov 2012;2:405-413.
130. Rosner $M$, Hengstschläger M. Targeting epigenetic readers in cancer. N Engl J Med 2012;367 1764-1765.

131. Wilkerson MD, Yin X, Hoadley KA et al. Lung squamous cell carcinoma mRNA expression subtypes are reproducible, clinically important, and correspond to normal cell types. Clin Cancer Res 2010;16:4864-4875.

132. Bergethon K, Shaw AT, Ou SH et al. ROS1 rearrangements define a unique molecular class of lung cancers. J Clin Oncol 2012;30:863-870.

133. Takeuchi K, Soda M, Togashi Y et al. RET, ROS1 and ALK fusions in lung cancer. Nat Med 2012;18: 378-381.

134. Kwak EL, Bang YJ, Camidge DR et al. Anaplastic lymphoma kinase inhibition in non-smallcell lung cancer. N Engl J Med 2010;363:16931703.

135. Shaw AT, Yeap BY, Mino-Kenudson M et al. Clinical features and outcome of patients with nonsmall-cell lung cancer who harbor EML4-ALK. J Clin Oncol 2009;27:4247-4253.

136. Carvalho RH, Haberle V, Hou J et al. Genome-wide DNA methylation profiling of nonsmall cell lung carcinomas. Epigenetics Chromatin 2012;5:9.

137. Tseng RC, Lee CC, Hsu HS et al. Distinct HIC1SIRT1-p53 loop deregulation in lung squamous carcinoma and adenocarcinoma patients. Neoplasia 2009;11:763-770

138. Furonaka $\mathrm{O}$, Takeshima $\mathrm{Y}$, Awaya $\mathrm{H}$ et al. Aberrant methylation of p14(ARF), p15(INK4b) and p16(INK4a) genes and location of the primary site in pulmonary squamous cell carcinoma. Pathol Int 2004;54:549-555.

139. Wistuba II, Gazdar AF. Lung cancer preneoplasia. Annu Rev Pathol 2006;1:331-348. 